\title{
Radiological simultaneous portohepatic vein embolization (RASPE) and major hepatectomy with hepatocellular carcinoma (HCC)
}

\author{
Georgios K. Glantzounis, Anastasia Karampa, Dimitra Peristeri \\ HPB Unit, Department of Surgery, University Hospital of Ioannina and School of Medicine, University of Ioannina, Ioannina, Greece \\ Correspondence to: Georgios K. Glantzounis, MD, PhD, FEBS. Professor of Surgery and Transplantation, Head of HPB Unit, Department of Surgery, \\ School of Medicine, University of Ioannina, 45 110, Ioannina, Greece. Email: gglantzounis@uoi.gr; gglantzounis@gmail.com. \\ Comment on: Laurent C, Fernandez B, Marichez A, et al. Radiological Simultaneous Portohepatic Vein Embolization (RASPE) Before Major \\ Hepatectomy A Better Way to Optimize Liver Hypertrophy Compared to Portal Vein Embolization. Ann Surg 2020;272:199-205.
}

Submitted Dec 30, 2020. Accepted for publication Feb 02, 2021.

doi: $10.21037 / \mathrm{hbsn}-20-865$

View this article at: http://dx.doi.org/10.21037/hbsn-20-865

Radiological simultaneous portohepatic vein embolization (RASPE) is a relatively new technique, which aims to rapidly increase the future liver remnant (FLR) in patients with very small FLR in order to undergo major hepatectomy.

It was initially described by Hwang et al. as a two stages procedure, which, included portal vein embolization (PVE) first, followed by embolization of right hepatic vein (HVE) after several days (1). They concluded that sequential application of PVE and HVE is safe and leads to a stronger compensatory regeneration of the FLR than does PVE alone. Recently, the process of simultaneous portohepatic vein embolization has been reported by Guiu et al. (2). Briefly the procedure was done under general anesthesia. The right $\mathrm{HV}$ (and accessory right $\mathrm{HV}$ when present) were accessed first. Then the distal part of the right $\mathrm{HV}$ was punctured under US and the right PV branch was assessed with US-guided technique. Embolization was conducted using a mixture of iodized oil and n-butyl-cyanoacrylate. It was concluded that simultaneous PVE and HVE induces safe and rapid hypertrophy of the FLR before right hepatectomy (2).

Furthermore, two very recent studies by Laurent et al. (3) and Guiu et al. (4), mainly with patients with liver metastases and inadequate FLR, showed that RASPE or liver venous deprivation (LVD) which is a similar term, is safe and induces faster and greater FLR, with better functional capacity in comparison to PVE, with less risk of post-operative liver failure. For this reason RASPE could be considered a safer "radiological associating liver partition and portal vein ligation" (ALPPS) for staged hepatectomy. $\quad 31$

It would certainly be interesting to explore the potential 32 applications of RASPE in patients with hepatocellular 33 carcinoma (HCC).

HCC is the $5^{\text {th }}$ most common cancer worldwide and the $3^{\text {rd }}$ most common cause of death. Despite the vaccination for hepatitis $B$ and the effective anti-viral treatment for hepatitis $\mathrm{B}$ and $\mathrm{C}$ the world incidence is increasing. This is mainly due to the increase in non-alcoholic fatty liver disease (NAFLD) and steatohepatitis which are the hepatic components of metabolic syndrome (5).

HCC is mainly developed in chronic liver disease, where there is hepatic steatosis, fibrosis or cirrhosis. Liver resection and liver transplantation are the main treatment options in order to achieve long term survival or cure. Liver transplantation offers very good results, but has many limitations, as it is usually applied in selected patients which mainly fulfil the Milano criteria (single tumor $<5 \mathrm{~cm}, 3$ tumors $<3 \mathrm{~cm}$ each). Liver resection is the treatment of choice for large HCCs with preserved liver function. Recent evidence suggests that liver resection can expand its indications as it can be applied even in advanced stages of the disease (multinodular HCCs, HCCs with limited macrovascular invasion) with satisfactory longterm results $(6,7)$. Furthermore, anatomic resections with broad surgical margins $(>1 \mathrm{~cm})$ provide better results, as HCC has a tendency to invade the small branches of portal vein, and to cause intrahepatic dissemination. However, major liver surgery is prohibited by the presence of chronic liver disease, which can have a significant impact on portal 
venous pressure and significantly decreases the capacity for liver regeneration.

The gold standard for patients with HCC and inadequate FLR is PVE. The main indications for PVE, in patients with HCC and presence of liver fibrosis or cirrhosis, are FLR $<40 \%$ when liver function is good (ICGR15 <10\%) and FLR $<50 \%$ when liver function is affected (ICGR15: 10-20\%) (8). However, PVE has limitations: it can not be applied effectively when FLR is very small $(<25 \%)$ and a long waiting period ( $>4$ weeks) is required before liver resection, in order that adequate hypertrophy of FLR can be achieved. Furthermore, segement IV embolization is technically very demanding, when an extended right hepatectomy is required.

In order to increase the regeneration of the FLR, the ideal method should be safe, induce rapid liver regeneration with good FLR functionality and be associated with low postoperative mortality.

ALPPS, which is the alternative of PVE in patients with small FLR, provides rapid liver regeneration but not with good functionality. A recent systematic review assessed the role of ALPPS in 176 patients with HCC and inadequate FLR. They concluded that ALPPS is safe and feasible to treat selected patients with initially unresectable HCC but with high 90-day mortality (17.6\%) and, as yet unclear oncological outcomes (9).

RASPE has the potential to overcome the disadvantages of PVE and ALPPS: it increases the FLR rapidly and effectively, with preservation of liver function (FLR-F) as expressed by the use of Technetium (99mTc) mebrofenin scintigraphy (4), is safe and with low post-operative mortality. Furthermore, embolization of middle HVE facilitates the performance of extended right hepatectomy.

The increase in regeneration rate vs. PVE could be due to several factors: embolization of the HVE could prevent persistent portal inflow and could reduce porto-portal collaterals. Furthermore, RASPE can increase liver injury since occlusion of HVE out-flow and simultaneous PVE could reduce the flow in the hepatic artery through the hepatic arterial buffer response.

There are, of course, several questions which have to be answered: what will be the effect of RASPE in patients with liver fibrosis or cirrhosis, where liver regeneration capacity is reduced? The majority of patients, where RASPE has been done, are patients with colorectal metastases, where liver is not fibrotic. Also, as the combination of TACE and PVE seems to be more effective in patients with large HCCs or satellite lesions (8), what will be the risks for the liver after sequential application of TACE and RASPE?

RASPE is also a technically demanding procedure and it still has to be shown if it is easily reproducible.

The randomized trial which is running for the effect of RASPE $v s$. PVE in patients with colo-rectal liver metastases should provide several answers, on the FLR changes at 3 weeks after the procedure (10). However, a similar trial should be conducted with patients with HCC, as the regeneration process is different in diseased liver.

Overall, RASPE has the potential to become the procedure of choice in patients with HCC and small FLR $(<25 \%)$.

\section{Acknowledgments}

Funding: None.

\section{Footnote}

Provenance and Peer Review: This article was commissioned by the editorial office of Hepatobiliary Surgery and Nutrition. The article did not undergo external peer review.

Conflicts of Interest: All authors have completed the ICMJE uniform disclosure form (available at https://hbsn. amegroups.com/article/view/10.21037/hbsn-20-865/coif). The authors have no conflicts of interest to declare.

Ethical Statement: The authors are accountable for all aspects of the work in ensuring that questions related to the accuracy or integrity of any part of the work are appropriately investigated and resolved.

Open Access Statement: This is an Open Access article distributed in accordance with the Creative Commons Attribution-NonCommercial-NoDerivs 4.0 International License (CC BY-NC-ND 4.0), which permits the noncommercial replication and distribution of the article with the strict proviso that no changes or edits are made and the original work is properly cited (including links to both the formal publication through the relevant DOI and the license). See: https://creativecommons.org/licenses/by-nc-nd/4.0/.

\section{References}

1. Hwang S, Lee SG, Ko GY, et al. Sequential preoperative ipsilateral hepatic vein embolization after portal vein embolization to induce further liver regeneration in 
patients with hepatobiliary malignancy. Ann Surg 2009;249:608-616.

2. Guiu B, Chevallier P, Denys A, et al. Simultaneous transhepatic portal and hepatic vein embolization before major hepatectomy: the liver venous deprivation technique. Eur Radiol 2016;26:4259-67.

3. Laurent C, Fernandez B, Marichez A, et al. Radiological Simultaneous Portohepatic Vein Embolization (RASPE) Before Major Hepatectomy A Better Way to Optimize Liver Hypertrophy Compared to Portal Vein Embolization. Ann Surg 2020;272:199-205.

4. Guiu B, Quenete F, Panaro Q, et al. Liver venous deprivation versus portal vein embolization before major hepatectomy: future liver remnant volumetric and functional changes. Hepatobiliary Surg Nutr 2020;9:564-76.

5. Anastasopoulos N-A, Lianos G, Tatsi V, et al. Clinical heterogeneity in patients with non-alcoholic fatty liver disease associated with hepatocellular carcinoma. Expert Rev Gastroenterol Hepatol 2020;14:1025-33.

6. Glantzounis K, Paliouras A, Stylianidi MC, et al. The role of liver resection in the management of intermediate and advanced stage hepatocellular carcinoma. A systematic review. Eur J Surg Oncol 2018;44:195-208.

Cite this article as: Glantzounis GK, Karampa A, Peristeri D. Radiological simultaneous portohepatic vein embolization (RASPE) and major hepatectomy with hepatocellular carcinoma (HCC). HepatoBiliary Surg Nutr 2021;10(2):267-269. doi: 10.21037/hbsn-20-865
7. Chen LT, Martinelli E, Cheng AL, et al. Pan-Asian 180 adapted ESMO Clinical Practice Guidelines for the 181 management of patients with intermediate and advanced/ 182 relapsed hepatocellular carcinoma: a TOSeESMO 183 initiative endorsed by CSCO, ISMPO, JSMO, KSMO, 184 MOS and SSO. Ann Oncol 2020;31:334-51. 185

8. Glantzounis GK, Tokidis E, Basourakos SP, et al. The role 186 of portal vein embolization in the surgical management of 187 primary hepatobiliary cancers. A systematic review. Eur J 188 Surg Oncol 2017;43:32-41.

9. Zhang J, Huang H, Bian J, et al. Safety, feasibility, and 190 efficacy of associating liver partition and portal vein 191 ligation for staged hepatectomy in treating hepatocellular 192 carcinoma: a systematic review. Ann Transl Med 193 2020;8:1246.

10. Deshayes E, Piron L, Bouvier A et al. Study protocol 195 of the HYPER-LIV01 trial: a multicenter phase 196 II, prospective and randomized study comparing 197 simultaneous portal and hepatic vein embolization to 198 portal vein mobilization for hypertrophy of the future 199 liver remnant before major hepatectomy for colo-rectal 200 liver metastases. BMC Cancer 2020;20:574. 